\title{
Perfil fitness en un grupo de estudiantes universitarios. Boyacá 2014
}

\section{Fitness profile in a group of university students. Boyacá 2014}

\author{
Yessica Ivonne Pérez Lemus ${ }^{1}$, Deisy Johanna Nonzoque Albaํ, Carolina Sandoval Cuellar
}

Forma de citar: Pérez Lemus YI, Nonzoque Alba DJ, Sandoval Cuellar C. Perfil fitness en un grupo de estudiantes universitarios. Boyacá 2014. Rev Univ Ind Santander Salud. 2016; 48(1): 51-59. DOI: http://dx.doi.org/10.18273/ revsal.v48n1-2016006 (c) (1)

\section{RESUMEN}

Introducción: La práctica de actividad física reduce el riesgo y previene enfermedades no trasmisibles, mejora la salud ósea y funcional, mantiene el equilibrio calórico y el control del peso, mejorando no sólo la condición física o perfil fitness, el cual se ve reflejado en la habilidad para llevar a cabo las tareas diarias con vigor y sin fatiga; en relación con la salud. Objetivo: Determinar el perfil fitness de estudiantes universitarios en el departamento de Boyacá. Metodología: Estudio descriptivo con diseño transversal, el cual contó con una población de 204 universitarios, de los cuales se obtuvo una muestra de 40 con el uso del software Epidat ${ }^{\circledR}$, con una confiabilidad del $95 \%$ y error estimado del $5 \%$, el muestreo fue estratificado con afijación proporcional. Se evaluaron los componentes fitness relacionados con la salud: capacidad aeróbica, fuerza muscular, flexibilidad y composición corporal. Se obtuvieron medidas de tendencia central, frecuencia y se aplicaron pruebas de asociación como Chi cuadrado, Pearson, Spearman y U de Mann-Whitney. Resultados: La edad promedio de la población fue 21,13 años (DS 2,79), el 67,5\% se clasifico según Índice de masa corporal en normopeso, el 35\% se ubicó en el rango obeso según su porcentaje graso, la fuerza muscular promedio para prensión manual fue de 30,02 Kilogramos (DS 12,85), miembros inferiores 70,48 Kilogramos (DS 40,88) y tronco 69,6 kilogramos (DS 38,5). El 85\% de las mujeres y el $81,8 \%$ de los hombres en la evaluación de la capacidad aeróbica se clasificaron en el rango muy pobre. Se reportan asociaciones estadísticamente significativas entre la capacidad aeróbica y el género ( p 0.00), la fuerza de tronco, miembro superior e inferior con el género con ( $\mathrm{p} 0.003$ ), $(\mathrm{p} 0,04)$ y $(\mathrm{p} 0,038)$ respectivamente. Conclusiones: La condición física o perfil fitness no es adecuado para la edad de los participantes teniendo en cuanta los bajos niveles de la fuerza muscular y la capacidad aeróbica obtenidos, así como el contar estudiantes en una alta frecuencia clasificados como obesos según su porcentaje graso.

Palabras clave: Composición corporal, fuerza muscular, sexo, aptitud física, salud.

1. Universidad de Boyacá. Tunja, Boyacá

Correspondencia: Carolina Sandoval Cuellar. Dirección: Carrera 2 Este No 64 -169. Edificio Uno. Oficina 106C. Correo electrónico: carolinasandoval@uniboyaca.edu.co. Teléfono: +87 450000 ext. 1108 


\section{ABSTRACT}

Introduction: Regular physical exercise prevents and reduces the risk of transmissible disease, improves functional bone health, maintains caloric balance and weight control, improving not only the physical condition or fitness profile, which is reflected in the ability to complete daily tasks with vigor and without fatigue; all relative to health. Objective: To determine the fitness profile of university students in the Department of Boyacá. Methodology: A descriptive study with cross-sectional design, which includes a population of 204 university students, of which a sample of 40 was obtained using Epidat ${ }^{\circledR}$ software, with $95 \%$ reliability and $5 \%$ error, the sampling was stratified with proportional allocation. The following fitness components were evaluated relative to health: aerobic capacity, muscular strength, flexibility and body composition. Measurements of central tendency and frequency were obtained, and association tests were applied such as Chi-squared, Pearson, Spearman and U Mann-Whitney. Results: The average age of the population was 21.13 years (SD 2.79), $67.5 \%$ were classified as normal weight under the Body Mass Index, 35\% were placed in the obese range based on percent body fat, the average muscular strength for hand grip was 30.02 kilograms (SD 12.85), 70.48 kilograms (SD 40.88) for lower extremities and 69.6 (SD 38.5) kilograms for core. $85 \%$ of women and $81.8 \%$ of men were classified in the very poor range when evaluating for aerobic health. Significant statistical association was reported between aerobic capacity and gender (p 0.00$)$, core strength, upper and lower extremities with gender at (p 0.0030, (p 0.04) and ( $p$ 0.038) respectively. Conclusions: The physical condition or fitness profile is not adequate for the age of the participants considering the low levels of muscular strength and aerobic capacity that were obtained, similar to the high frequency of students classified as obese according to body fat percentage.

Keywords: Body composition, muscle strength, sex, physical fitness, health

\section{INTRODUCCIÓN}

La condición física, también denominada como forma física o perfil fitness, destaca la capacidad o potencial físico de una persona en relación con la salud, estáligada a la demostración de rasgos y capacidades, asociadas a un bajo riesgo de desarrollar enfermedades hipocineticas o generar incapacidad funcional ${ }^{1}$. El Consejo Presidencial sobre Fitness, Deportes y Nutrición, (The Presidents Council on Physical Fitness and Sports - PCFSN) la define como la habilidad para llevar a cabo las tareas diarias con vigor y vigilancia, sin fatiga y energía suficiente para disfrutar de las actividades de tiempo libre y afrontar situaciones inesperadas, según Plasencia y Bolívar $^{2}$, de ahí que la práctica de actividad física entendida ésta, como cualquier movimiento corporal que causa un aumento en el gasto energético, la cual implica un movimiento planificado o estructurado del cuerpo realizado de manera sistemática en términos de frecuencia, intensidad y duración, y que está diseñado para mantener o mejorar los resultados relacionados con la salud ${ }^{3}$, así mismo, el ejercicio físico entendido como una actividad física planificada, estructurada y repetitiva que tiene por objetivo mejorar o mantener los componentes de la forma física ${ }^{4}$ éstas mejoran no sólo la condición física, sino que además tienen beneficio directo sobre las modificaciones fisiológicas que se dan como resultado de su práctica como parte de un estilo de vida dado desde la niñez hasta la adultez y vejez, lo que permitiría no sólo conservar una condición física 52 saludable, sino también un mejor rendimiento físico, emocional, intelectual y socio afectivo ${ }^{5}$.

Es importante resaltar que el perfil fitness debe tener en cuenta la capacidad funcional, siendo necesaria durante el desarrollo y ejecución de actividades de la vida diaria, además debe analizar cada una de las manifestaciones sobre la capacidad aeróbica, flexibilidad, fuerza muscular y las características antropométricas dando paso a un adecuado estado de salud, cabe resaltar que el elemento central de las relaciones entre la actividad física y la salud es la condición física ${ }^{5}$.

Bajo la conceptualización de Bouchard y Shepard, existe una relación directa entre salud y condición física, plasmada desde el Modelo de Toronto de Condición Física, Actividad Física y Salud, el cual muestra, que el nivel de la condición física está influenciado por la cantidad y tipo de actividad física realizada habitualmente, llegando ésta a modificar los niveles de práctica de actividad física, mejorando el estado de salud, y permitiendo al individuo ser más activo, así como contar con componentes adecuados de la condición física en relación con la salud como, la resistencia cardiorrespiratoria, resistencia muscular, fuerza muscular, composición corporal y la flexibilidad; Dichos autores afirman que si desde la niñez y durante la adolescencia y la adultez se mantiene una adecuada aptitud, los componentes del perfil fitness se encontraran en óptimas condiciones de funcionamiento 
durante la vejez, llevando esto, a un excelente estado de salud ${ }^{2}$. Lo expuesto anteriormente enmarca el interés de las investigadoras en relación con la descripción del perfil fitness de los estudiantes de Fisioterapia de la Universidad de Boyacá 2014.

\section{MATERIALES Y MÉTODOS}

La investigación se desarrolló bajo un enfoque cuantitativo, con un estudio tipo descriptivo y diseño transversal. La población fue 204 estudiantes de Fisioterapia, para la obtención de la muestra se hizo uso del software Epidat ${ }^{\circledR}$, los investigadores determinaron un nivel de confianza del $95 \%$, error estimado del $5 \%$, y con una desviación estándar de referencia según lo reportado el estudio "Perfil de Fitness de los estudiantes de la Universidad de Caldas en el 2009"6 (17.8 número de repeticiones/minuto), estableciendo una muestra de 40 individuos.

Se empleó un muestreo probabilístico estratificado proporcional según el semestre, obteniéndose una fracción muestral para conocer el número de individuos a evaluar en cada semestre, como se observa en la Tabla 1, una vez obtenida la muestra estratificada, los participantes se escogieron a través de un muestreo aleatorio simple con el uso del software Epidat ${ }^{\circledR}$.

Tabla 1. Muestreo estratificado con afijación proporcional.

\begin{tabular}{cccc}
\hline Semestre & $\begin{array}{c}\text { No. } \\
\text { estudiantes }\end{array}$ & wi & $\begin{array}{c}\text { Afijacion } \\
\text { Proporcional }\end{array}$ \\
\hline 1 & 41 & 0.20098039 & 8 \\
2 & 23 & 0.11274510 & 5 \\
3 & 36 & 0.1764706 & 7 \\
4 & 37 & 0.1813725 & 7 \\
5 & 18 & 0.0882353 & 4 \\
6 & 14 & 0.0686275 & 3 \\
7 & 14 & 0.0686275 & 3 \\
8 & 15 & 0.0735294 & 3 \\
9 & 6 & 0.0294118 & 1 \\
POBLACION & 204 & & 40 \\
MUESTRA & 40 & & \\
\hline
\end{tabular}

Para llevar a cabo la investigación a cada participante se le informó sobre el objetivo del estudio y sus posibles riesgos, complicaciones o efectos secundarios tras las pruebas realizadas, se tuvieron en cuenta criterios de inclusión como ser físicamente apto para la prueba, a partir, del diligenciamiento del formato Cuestionario de Preparación de Actividad Física (Physical Activity Readiness Questionnaire) - Par-Q; no haber consumido licor, trasnochado y no haber realizado ninguna actividad vigorosa el día antes de la prueba.

Se estableció como criterio de exclusión el tener una patología o enfermedad que impidiera el desarrollo del conjunto de pruebas a realizar. Una vez seleccionado el sujeto, se procede al diligenciamiento del consentimiento informado, posteriormente, se evaluaron variables antropométricas como la talla, medida con estadiómetro de pared SECA en centímetros, el peso en kilogramos y el porcentaje graso, que fueron medidos con la báscula Fit $\operatorname{Scan}^{\circledR}$ Body Fat Monitor BF-679F, este último, para clasificar a los participantes según la tabla propuesta por el Consenso $\mathrm{SEEDO}^{7}$, para la evaluación del sobrepeso y la obesidad, y cualidades físicas como, la fuerza muscular, definida como la capacidad de un grupo muscular para desarrollar una fuerza contráctil máxima contra una resistencia en una sola contracción ${ }^{8}$; se empleó la dinamometría de miembro superior, inferior y tronco con el dinamómetro Baseline ${ }^{\circledR}$ BackLeg-Chest Dynamometer, la flexibilidad, a su vez definida como el óptimo desempeño de la capacidad miotendinosa y articular para realizar movimientos de máxima amplitud posible, abarcando tres componentes tales como; la movilidad articular, la elasticidad y elongación musculo-ligamentosa ${ }^{9}$, se evaluó con la prueba de Sit and Reach modificada, finalmente, la capacidad aeróbica se midió con el test de BalkeWare, en caminadora marca Sportop ${ }^{\circledR}$ i Pro, previa calibración de los instrumentos.

Se tabularon los datos en el programa estadístico SPSS versión $20.0^{\circledR}$, donde, se calcularon medidas de tendencia central y de variabilidad o dispersión para variables cuantitativas incluidas en el estudio. En cuanto al análisis bivariado, se determinó la significancia estadística de las posibles relaciones resultantes con el uso del Chi cuadrado de Pearson establecidas a partir de las características propias de las variables categóricas (ordinales y nominales). Adicionalmente, se aplicó pruebas de normalidad a través de la prueba de Shapiro - Wilk y se establecieron las relaciones de variables numéricas a través de la aplicación de pruebas paramétricas como Pearson y no paramétricas como Spearman y U de Mann-Whitney.

La prueba de Shapiro - Wilk, estableció una distribución normal para el peso, la talla y el $\mathrm{VO}_{2}$ máx, por lo cual se utiliza el método de correlación paramétrico de Pearson. En cuanto, a las variables cuya distribución no fue normal con $p<0.05$, como la edad, la dinamometría 
de miembro superior, de miembro inferior y de tronco se aplica el método para correlación no paramétrico de Spearman y U de Mann-Whitney.

Una vez terminado el proceso de evaluación y trabajo de campo, a los sujetos participantes se les informó de manera general sobre sus cualidades físicas. El proyecto de investigación fue aprobado por el Comité de Investigación de Facultad Ciencias de la Salud de la Universidad de Boyacá.

\section{RESULTADOS}

Fueron evaluados 40 estudiantes del programa de fisioterapia de la Universidad de Boyacá en el II periodo del año 2013 donde el promedio de la edad fue de 21,13 años DS 2,79 años, con edad mínima de 16 años y máxima de 32 años, el rango de estatura estuvo entre $143 \mathrm{~cm}$ y $185 \mathrm{~cm}$ con un promedio de $161,98 \mathrm{~cm}$, DS $8,94 \mathrm{~cm}$; y el peso corporal oscilo entre 37 y $80 \mathrm{~kg}$.

Tabla 2. Medidas de Tendencia central de la población objeto (n: 40).

\begin{tabular}{lcccccc}
\hline & MEDIA & $*$ DS & $* *$ IC 95\% & MEDIANA & $* * *$ MIN & $* * *$ MAX \\
\hline Edad Años & 21,13 & 2,79 & $20,23-22,2$ & 21,00 & 16 & 32 \\
Peso Kg. & 56,88 & 10,4 & $53,53-60,22$ & 54,60 & 37 & 80 \\
Talla Cm. & 161,98 & 8,94 & $159,11-164,84$ & 161 & 143 & 185 \\
Dinamometría Miembro superior Kg. & 30,02 & 12,85 & $25,91-34,14$ & 26,00 & 10 & 65 \\
Dinamometría Miembro inferior Kg. & 70,48 & 40,88 & $57,40-83,55$ & 62,5 & 20 & 187 \\
Dinamometría de tronco Kg. & 69,61 & 38,5 & $57,29-81,94$ & 60,0 & 25 & 198 \\
VO2Máx. ml.kg.min & 17,02 & 8,4 & $14,32-19,71$ & 15,75 & 2,20 & 38,8 \\
\hline
\end{tabular}

*DS: Desviación estándar. **IC 95\%: Intervalo del confianza al 95\%.***MIN: Valor mínimo. ****MAX: Valor máximo.

En relación al género, el $82,5 \%$ eran mujeres y $17,5 \%$ hombres, referente al IMC el 67,5\% (n=27) son clasificados en la categoría de normopeso, seguido de 6 individuos con bajo peso y 6 con sobrepeso con un porcentaje de $15 \%$ respectivamente, por último, se presentó obesidad tipo I en el $2,5 \%$ de la muestra.

Al clasificar el porcentaje graso según el referente del Centro para la Investigación para la Obesidad de New York $^{10}$, se obtuvo que 12 individuos correspondientes al $35 \%$ presentaron porcentaje graso saludable, seguido de bajo en grasa con un $27,5 \%$ que correspondió a 11 individuos, en la clasificación obeso se ubican con el 22,5\% 8 individuos, a su vez en alto en grasa 6 individuos con un $15 \%$, y por último, en obesidad tipo 1 con 3 individuos el $8 \%$ restante. Tabla 2.

Al determinar la fuerza muscular de miembro superior mediante la fórmula (peso presionado en Kilogramos/ peso corporal en kilogramos) según el Manual de la condición física relacionado con la salud (Health Related Physical Fitness Assessment Manual 2002) del Colegio Americano de Medicina del Deporte
(American College of Sports Medicine ACSM's) ${ }^{11}$, se obtuvo un promedio de prensión manual de 30,02 $\mathrm{Kg}$. DS 12,85 Kg., donde el valor mínimo de prensión fue de $10 \mathrm{Kg}$. y el máximo de $65 \mathrm{Kg}$., asimismo, al evaluar la fuerza en miembros inferiores se obtuvo un promedio de $70,48 \mathrm{Kg}$. DS 40,88 Kg.; con un valor mínimo en fuerza realizada por miembros inferiores de $20 \mathrm{Kg}$. y un máximo de $187 \mathrm{Kg}$., clasificándose la mayoría de la población en rango muy pobre, adicionalmente, se reportó en fuerza de tronco, un promedio de 69,6 kg. DS 38,5 kg; con un mínimo de fuerza realizada por tronco de $25 \mathrm{~kg}$. y un máximo de $198 \mathrm{~kg}$. clasificándose la mayoría de la población en rango muy pobre tanto para el género femenino como masculino. Tabla 2.

Al determinar la flexibilidad en estudiantes participantes, se evidenció que el $40 \%$ se clasificó en rango excelente, el $27,5 \%$ en rango malo, seguido del rango aceptable y bueno con un $15 \%$, y por último, 1 individuo en calificación deficiente correspondiente al $2 \%$, valores determinados según clasificación del Manual Evaluación de Aptitudes Físicas ${ }^{12}$. Tabla 3. 
Tabla 3. Distribución de frecuencias de las variables categóricas del objeto de estudio (n: 40)

\begin{tabular}{|c|c|c|c|c|}
\hline & Clasificación & $\begin{array}{c}\text { Frecuencia } \\
\text { Absoluta }\end{array}$ & $\begin{array}{c}\text { Frecuencia } \\
\text { Relativa }\end{array}$ & $\begin{array}{c}\text { IC } \\
95 \%\end{array}$ \\
\hline \multirow{2}{*}{ Género } & Hombres & 7 & 17,5 & $0.5-28$ \\
\hline & Mujeres & 33 & 82,5 & $71-94$ \\
\hline \multirow{4}{*}{ IMC } & Bajo peso & 6 & 15,0 & $0.3-26$ \\
\hline & Normo peso & 27 & 67,5 & $5-81$ \\
\hline & Sobrepeso & 6 & 15,0 & $0.3-26$ \\
\hline & $\begin{array}{l}\text { Obesidad } \\
\text { tipo } 1\end{array}$ & 1 & 2,5 & $15-44$ \\
\hline \multirow{5}{*}{$\%$ Graso } & Bajo en grasa & 11 & 27,5 & $13-40$ \\
\hline & Saludable & 12 & 35,0 & $15-44$ \\
\hline & Alto en grasa & 6 & 15,0 & $0.3-23$ \\
\hline & Obeso & 8 & 22.5 & $0,7-32$ \\
\hline & $\begin{array}{l}\text { Obesidad } \\
\text { tipo } 1\end{array}$ & 3 & 8 & $-0.4-16$ \\
\hline \multirow{5}{*}{ Flexibilidad } & Mala & 11 & 27,5 & $14-41$ \\
\hline & Deficiente & 1 & 2,5 & $0,7-32$ \\
\hline & Aceptable & 6 & 15,0 & $0.3-26$ \\
\hline & Buena & 6 & 15,0 & $0.3-26$ \\
\hline & Excelente & 16 & 40,0 & $24-55$ \\
\hline
\end{tabular}

Por último, el comportamiento de la capacidad aeróbica de los estudiantes según la clasificación para las pruebas y prescripción del ejercicio Guidelines for Exercise Testing and Prescriptión del Colegio Americano de Medicina del Deporte (American College of Sports Medicine ACSM's) ${ }^{11}$, mediante el protocolo de Balke, muestra mayor frecuencia en un rango muy pobre en un $85 \%$ y $81,8 \%$ en hombres y mujeres respectivamente, seguido de la categoría pobre con un $15 \%$ en hombres y $15,2 \%$ en mujeres, solo el $3 \%$ de las mujeres se ubicó en rango bueno Tabla 4. El promedio del consumo de $\mathrm{VO}_{2}$ fue de 17,02 ml.kg.min. DS 8,4 ml.Kg.min. Tabla 2.

La asociación de las variables capacidad aeróbica y genero mediante prueba del coeficiente Chi Cuadrado de Pearson, evidencia asociación significativa ( $p 0.00)$ Tabla 4. Para las variables IMC, porcentaje graso, flexibilidad, para el género los valores reportados no establecieron asociaciones significativas.

Respecto a la correlación entre el peso y la talla con el $\mathrm{VO}_{2}$ máx, se reportan valores según el coeficiente de correlación de Pearson de 0,020 y valor $p 0.903$ y 0,300 , y valor $p 0,060$ respectivamente, estableciendo una correlación insignificante negativa, según Makuka ${ }^{13}$.

Tabla 4. Correlación de variables género y explicativas

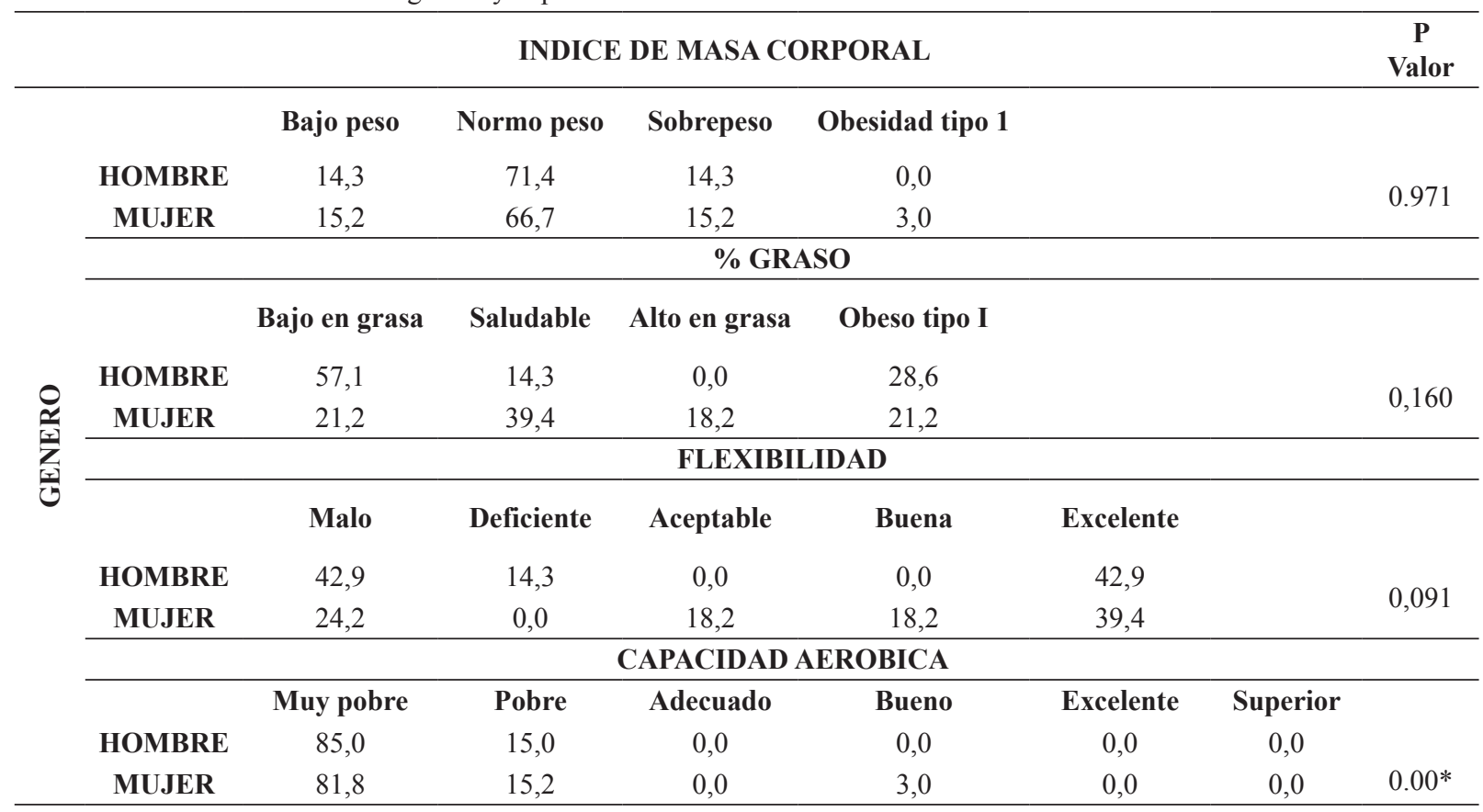

* Significancia estadística $p<0,05$.

Continuando con las variables cuya distribución no fue normal, se correlacionaron la dinamometría de miembro superior y la edad obteniéndose un valor del coeficiente de correlación de Spearman de 0,136, y valor $p 0.40$, este comportamiento fue similar para la dinamometría de miembro inferior y de tronco con la edad, donde se 
obtuvieron valores del coeficiente de correlación de Spearman de 0,21 , y valor $p 0,19$ y 0,24 y valor $p 0,38$ respectivamente.

Se evidencio así, una correlación significativa entre la fuerza de tronco, de miembro superior e inferior con el género con valores $p 0.003, p 0,04$ y $p 0,038$ respectivamente obtenidas con la U de Mann - Whitney.

\section{DISCUSIÓN}

La evaluación de los componentes del perfil fitness realizado en la presente investigación evidenció una mayor población femenina. Referente a la composición corporal, la variable talla tuvo valores mínimos y máximos respectivamente de 143 y 185 centímetros, valor que al ser contrastado con el reportado por la investigación denominada Evaluación Antropométrica de Estudiantes Universitarios en Bogotá, Colombia, la cual determinó un valor mínimo de estura de $142 \mathrm{~cm}$ y el máximo $183 \mathrm{~cm}^{14}$, evidencia un comportamiento similar en dicha variable.

Al determinar el IMC, los individuos reportaron niveles por debajo del promedio nacional según Encuesta Nacional de Salud (ENSIN) para personas entre 18 a 64 años de edad. Según la encuesta realizada en el 2010 en el departamento de Boyacá, se encuentra que la mayor frecuencia de individuos se ubicaron en el rango normo peso, de acuerdo a la clasificación de la $\mathrm{OMS}^{15}$, dato concordante con los valores obtenidos por Montero et al. ${ }^{8}$; sin embargo, según la investigación realizada por Martínez ${ }^{16}$, estos valores se alejan, atendiendo a que se encontró que el $28,5 \%$ y el $14,2 \%$ de hombres y mujeres, respectivamente, presentaron sobrepeso. Estos resultados pueden diferir atendiendo a que los porcentajes fueron obtenidos con el uso de medidas antropométricas, mencionada por la mayoría de estudios sobre composición corporal, donde se miden pliegues cutáneos, no obstante, existen evidencias que muestran una correlación con el porcentaje graso obtenido a través de diversas técnicas como la densitometría ${ }^{16}$; método que fue utilizado en la presente investigación, a través de la báscula Fit Scan ${ }^{\circledR}$ Body Fat Monitor BF-679F, lo anterior sustentado en estudios más recientes los cuales han demostrado su efectividad a la hora de determinar esta variable, basándose en la teoría de derivación de una nueva ecuación de predicción para el cálculo de la composición corporal, realizada propiamente por el equipo, de ahí que, el procedimiento se basa en incorporar datos como el sexo, la edad, y una transformación logarítmica de la altura, además, el peso y la impedancia medida por medio de la ecuación desarrollada por la báscula anteriormente mencionada $^{14}$. Al comparar los resultados del estudio de Vargas et al. ${ }^{14}$, donde hallaron que el $60,4 \%$ de los individuos participantes se encontraban dentro de los parámetros normales para el área grasa del brazo, mientras que el $16,6 \%$ presentó exceso de reserva grasa, de acuerdo con el percentil como indicador, discriminando por género, se determinó la ubicación de los hombres entre los percentiles 10 y 25 clasificado como inadecuado que al compararlos con los de las mujeres se ubicaron entre los percentiles 25 y 50 clasificados como adecuado ${ }^{14}$; al hacer el uso del instrumento directo, la báscula Fit Scan ${ }^{\circledR}$ Body Fat Monitor BF-679F, se halló que el 62,5\% se sitúa en el parámetro normal y el $37,5 \%$ represento exceso de grasa, valores que se alejan de los obtenido por el estudio de Vargas et al. ${ }^{14}$

Otro componente del fitness corporal, que se ha considerado como un elemento básico y determinante del rendimiento físico y humano es la fuerza que según la clasificación del Manual de la condición física relacionado con la salud (Health - Related Physical Fitness Assessment) Manual 2002 del Colegio Americano de Medicina del Deporte (American College of Sports Medicine ACSM's 2002) ${ }^{11}$, evidencia para el presente estudio un valor mínimo de prensión manual 10 Kilogramos y máximo de 65 Kilogramos, de igual manera, al evaluar la fuerza en miembros inferiores se obtuvo que el valor mínimo de fuerza realizada fue de 20 Kilogramos y máximo de 187 Kilogramos, por otro lado, en cuanto a la fuerza de tronco, el mínimo de fuerza fue de 25 kilogramos y el máximo de 198 kilogramos, lo anterior calificó a los individuos en el rango de muy pobre tanto para el género femenino como masculino, sin embargo, los datos obtenidos estuvieron por debajo de la clasificación respecto al estudio realizado por Ramos et al. ${ }^{6}$, quienes encontraron que en ambos sexos el promedio de la fuerza manual realizada se halló en la categoría regular, adicionalmente, se evidencia que en los estudios citados no se tuvo en cuenta la evaluación de fuerza muscular en tronco y miembro inferior mencionados por Sandoval ${ }^{12}$.

Alba $^{17}$ en la investigación del Perfil Fitness de los estudiantes de la Universidad de Caldas, referente a la flexibilidad, encontró que los promedios de ambos sexos se ubican en la categoría media, clasificación que se aleja según los resultados obtenidos en el presente estudio donde el $55 \%$ se ubica en categoría excelente, según Heyward ${ }^{11}$. Por otro lado, respecto a lo planteado por Neiva et al. ${ }^{18}$, en su estudio "Perfil de la aptitud 
física de los practicantes de Parkour" estableció como clasificación para la flexibilidad aptitud baja en un $69,23 \%$ de la muestra.

Al determinar el comportamiento de la capacidad aeróbica con la utilización del protocolo de Balke-Ware ${ }^{19}$ como método indirecto, se estableció una clasificación muy pobre; valor similar al ser contrastado con lo evidenciado en el estudio de Neiva et al. ${ }^{18}$, donde la capacidad aeróbica se clasificó como "baja capacidad", y tan solo el $46.1 \%$ tuvo la capacidad cardiorrespiratoria adecuada. Similitud también encontrada por Secchi et al. ${ }^{20}$, donde determinaron que la población se clasificó con un nivel de capacidad aeróbica no saludable.

Al establecer la correlación entre el IMC, porcentaje graso, flexibilidad y el género no se encontró asociación significativa, es decir, que el género no influye sobre el comportamiento de los componentes del perfil fitness, otros autores como Doriot y Wang ${ }^{21}$, mencionan que el efecto del género es más débil que la edad. Adicionalmente, mencionan referente al porcentaje graso y el género que la diferencia radica en que las mujeres tienen mayor grasa corporal, concomitantemente tienden a ser físicamente menos activas, esto asociado a factores socioculturales, lo cual incrementa con el trascurso de la adolescencia, sin embargo, estas evidencias científicas son contrarias a lo expuesto por Escudero et al. ${ }^{22}$, quienes al evaluar el componente antropométrico de 150 jugadores de voleibol, reportaron que los grupos sub Femenino, sub Femenino y Absoluto Femenino, obtuvieron menor tejido graso en el grupo Absoluto y los valores más altos del componente graso se encontró en el grupo Sub 21 Femenino, en cuanto, a la Sub 19 Femenino en comparación a la Sub 21 presento mayor porcentaje graso, lo que demuestra que el componente de porcentaje graso es menor en proporción en grupos avanzados.

Carrasco et al. ${ }^{23}$ mencionan que el IMC puede inducir a errores en la predicción a nivel individual, puesto que al hablar de esta variable en relación al género, se ha demostrado, especialmente que en hombres a pesar de que son clasificados como obesos con el cálculo de un IMC igual o mayor de $30 \mathrm{~kg} / \mathrm{m}^{2}$, presentan un porcentaje de masa grasa menor al valor sugerido, clasificándolos como "falsos positivos". Esta discrepancia entre el IMC y el porcentaje graso puede estar relacionada con el nivel de actividad física o con componentes etnográficos, lo anterior hace referencia a que en sujetos con un mayor nivel de actividad física, lleva al aumento en la masa magra, a la vez contribuye parcialmente al exceso de peso y a un mayor índice de masa corporal. La mayor frecuencia $67,5 \%$ de los individuos se ubicó en el IMC normopeso, no obstante, el $39,4 \%$ las mujeres presentaron niveles altos en los porcentajes graso, en forma contraria los hombres en un $71,4 \%$ tuvieron niveles bajos de grasa.

Al determinar la correlación entre la dinamometría de miembro superior, inferior y tronco con la edad y el género se obtuvo correlación de estas variables; correlación no reportada en el estudio de Rojas et al. ${ }^{18}$, por otro lado, esta correlación significativa también fue reportada por Williams et al., donde encontraron que la fuerza muscular, el género y la edad tuvieron valores de $p<0,001^{24}$.

Escalona et al. ${ }^{25}$, mencionan que en mujeres y hombres la fuerza de prensión hasta los 12 años no muestra diferencias, sin embargo, luego de este rango de edad el aumento depende de diferencias estructurales y bioquímicas de origen hormonal, que se contrastan con factores culturales y llevan a que el hombre realice tareas más dirigidas al desarrollo de la fuerza del crecimiento corporal, aumento de masa muscular, número de fibras musculares y velocidad de contracción existentes anatómicamente, estableciendo el número y grosor de las miofibrillas desarrolladas.

Respecto a la capacidad aeróbica se halló asociación significativa con el género, datos que concuerdan con los hallados por Shaiza et al. ${ }^{26}$ que al evaluar la capacidad aeróbica en mujeres con sobrepeso y mujeres de peso normal hallaron que el $\mathrm{VO}_{2}$ máx. en los grupos 1 y 2 fue $34,52 \pm 3,26 \mathrm{ml} / \mathrm{min} / \mathrm{kg}$ y $37,51 \pm 2,88$ $\mathrm{ml} / \mathrm{min} / \mathrm{kg}$, respectivamente y concluyeron que la diferencia en el $\mathrm{VO}_{2}$ máx. encontrado en las mujeres con sobrepeso fue estadísticamente significativa con valor de p $0,002^{26}$, de igual forma, Kostrzewa et al. ${ }^{27}$, determinaron el $\mathrm{VO}_{2}$ máx. en 22 mujeres jóvenes divididas en tres grupos: bajo peso, peso normal y sobrepeso encontrando consumo máximo de oxígeno de $2,49 \mathrm{ml} / \mathrm{kg} / \mathrm{min}$; con un valor $p 0,047$, se observó entre las mujeres de bajo peso.

Zarrazquin et al. ${ }^{28}$, encontró que los valores de capacidad aeróbica en estudiantes de ciencias de la salud estaba dentro del rango normal, pero el $\mathrm{V0}_{2}$ máx. durante el primer y segundo año de estudios disminuyó con un valor $p<0,05$, en los hombres disminuyó desde el principio hasta el final del estudio, mientras que en mujeres disminuyó entre el primer y segundo año.

Estudios han demostrado que hombres y mujeres con mayor capacidad aeróbica tienen menor cantidad 
de masa grasa total; cabe resaltar que los jóvenes universitarios entran en un periodo crítico en sus vidas al ingresar a la educación superior, debido a que tienden a disminuir su nivel de actividad física y por tanto aumentan su peso, adicionalmente, adquieren prácticas de conductas para controlar el peso, tales como utilizar dietas inadecuadas que favorecen el riesgo tanto a desórdenes alimentarios como de obesidad y sobrepeso afectando la capacidad aeróbica como indicador de condición física y su relación con el estado nutricional ${ }^{20}$.

\section{CONCLUSIONES}

Los individuos presentaron un peso adecuado para la estatura, sin embargo, un alto número de participantes se clasificó en porcentajes grasos altos, obesos y obesidad tipo de acuerdo a la clasificación de la OMS.

La fuerza de prensión manual, miembros inferiores y tronco, así como la capacidad aeróbica en los participantes en este estudio se calificaron en rango muy pobre tanto para el género femenino como masculino, comportamiento diferente respecto a la flexibilidad, pues este componente del fitness corporal en la población objeto tuvo una calificación de adecuada.

Finalmente, los resultados hallados en la determinación del perfil fitness de los estudiantes de Fisioterapia de la Universidad de Boyacá, demuestran que los jóvenes presentan bajos niveles en los componentes del fitness, por lo cual el grupo de investigadores puede llegar a inferir sobre los inadecuados estilos de vida y la baja practica de actividad física. Adicionalmente, expone la disyuntiva acerca del proceso de apropiación en su formación de la importancia sobre el adecuado estado de los componentes del perfil fitness en relación a la salud.

\section{AGRADECIMIENTOS}

Alos docentes y estudiantes del Programa de Fisioterapia de la Universidad de Boyacá. Financiación: artículo producto de un proyecto de investigación financiado por la Universidad de Boyacá entidad que no influyó en el diseño del estudio.

\section{CONFLICTO DE INTERÉS}

Los autores declaran no tener conflictos de interés.

\section{REFERENCIAS}

1. American College of Sports Medicine. Manual para la valoración y prescripción del ejercicio. 2a ed. Badalona, España: Paidotribo; 2005: p. 26-45.

2. Tammelin T. Physical activity from adolescence to adulthood and health-related fitness at age 31 . Cross-sectional and longitudinal analyses of the Northern Finland birth cohort of 1966. Oulu, Finland: Department of Public Health Science and General Practice, University of Oulu; 2003.

3. Meneses J, González E, Correa J, Ramírez R. Revisión Intervenciones con actividad física supervisada en el manejo de la fatiga relacionada con el cáncer: una revisión sistemática. Nutr Hosp. 2014; 30(3): 486-497.

4. Firman GO. Fisiología del ejercicio Físico. Corrientes, Argentina: Facultad de Medicina de la UNNE. 2000.

5. Devís J, Peiró C. La actividad física y la promoción de la salud en niños/as y jóvenes: la escuela y la educación física. Rev Psicol Deport. 1993; 2(2): 71-86.

6. Ramos S, Álzate DA, Ayala JE, Franco AM, Sánchez JM. Perfil de fitness de los estudiantes de la Universidad de Caldas. Hacia Promo Salud. 2009; 14(1): 23-34

7. Rubio MA, Salas Salvadó J, Montserrat B, Moreno B, Aranceta J, Bellido D, et al. Consenso SEEDO 2007 para la evaluación del sobrepeso y la obesidad y establecimiento de criterios de intervención terapéutica. Rev Esp Obes. 2007; 5(3): 135-175. DOI:10.1016/S0025-7753(07)72531-9.

8. Montero Bravo A, Úbeda Martín N, García González A. Evaluation of dietary habits of a population of University students in relation with their nutritional knowledge. Nutr Hosp. 2006; 21(4): 466-473.

9. Ortega R. Medicina del ejercicio físico y el deporte. 2 ed. Madrid, España: Díaz de Santos; 2000.

10. Gallagher D, Heymsfield SB, Heo M, Jebb SA, Murgatroyd PR, Sakamoto Y. Healthy percentage body fat ranges: an approach for developing guidelines based on body mass index. Am J Clin Nutr. 2000; 72(3): 694-701.

11. Heyward V. Evaluación de la aptitud física y prescripción del ejercicio. 5 ed. Madrid, España: Médica Panamericana; 2006.

12. Sandoval C. Manual evaluación de aptitudes físicas. Tunja, Boyacá: Universidad de Boyacá; 2009.

13. Mukaka MM. A guide to appropriate use of Correlation coefficient in medical research. Malawi Med J. 2012; 24(3): 69-71. 
14. Vargas M, Becerra F, Prieto E. Evaluación antropométrica de estudiantes Universitarios en Bogotá, Colombia. Rev Salud Pública. 2008; 10(3): 433-442.

15. Colombia. Ministerio de la Protección Social. Encuesta nacional de la situación nutricional en Colombia 2010. Bogotá: Instituto Colombiano de Bienestar Familiar; 2009.

16. Martínez C, Veiga P, López de Andrés A, Cobo JM, Carbajal A. Evaluación del estado nutricional de un grupo de estudiantes universitarios mediante parámetros dietéticos y de composición corporal. Nutr Hosp. 2005; 20(3): 197-203.

17. Alba A. Test Funcionales, cineantropometría y prescripción del entrenamiento. 2 ed. Armenia: Kinesis; 2006.

18. Neiva L, Pinto R, Cieslakl F, Ishiyama M, Milano $\mathrm{G}$, Stefanello J. Physical fitness profile of le parkour practitioners. Rev Bras Med Esporte. 2011; 17(3): 198-201.

19. López CJ, Fernández VA. Fisiología del ejercicio. 3 ed. Buenos Aires: Médica Panamericana; 2008.

20. Secchi JD, García GC. Aptitud física cardiorrespiratoria y riesgo cardiometabólico en personas adultas jóvenes. Rev Esp Salud Publica. 2013; 87(1): 35-48.

21. Atkinson G, Nevill AM. Statistical methods for assessing measurement error (reliability) in variables relevant to sports medicine. Sports Med. 1998; 26(4): 217-238.

22. Ramirez Lechuga J, Muros Molina J, Morente Sánchez J, Sánchez Muñoz C, Femia Marzo P,
Zabala Díaz M. Effect of an 8-week aerobic training program during physical education lessons on aerobic fitness in adolescents. Nutr Hosp. 2012; 27(3): 747-754. DOI: 10.3305/nh.2012.27.3.5725.

23. Carrasco F, Reyes E, Rimler O, Ríos F. Exactitud del índice de masa corporal en la predicción de la adiposidad medida por impedanciometría bioeléctrica. Arch Latinoam Nutr. 2004; 54(3): 280-286.

24. Andrews AW, Thomas MW, Bohannon RW. Normative values for isometric muscle force measurements obtained with hand-held dynamometers. Phys Ther. 1996; 76(3): 248-259.

25. Escalona P, Naranjo J, Lagos V, Solís F. Parámetros de normalidad en fuerzas de prensión de mano en sujetos de ambos sexos de 7 a 17 años de edad. Rev Chil Pediatr. 2009; 80(5): 435-443.

26. Shazia SM, Badaam Km, Deore DN. Evaluación de aeróbica capacidad en sobrepeso jóvenes mujeres: un estudio transversal. Int J Appl Básica Med Res. 2015; 5(1): 18-20.

27. Kostrzewa ND, Nowak R, Jastrzębski Z, Zarebska A, Bichowska M, Drobnik KI, et al. Efecto de 12 semanas de duración de un programa de capacitación sobre la composición corporal, capacidad aeróbica, hemograma completo y el perfil lipídico en sangre entre jóvenes mujeres. Biochem Med. 2015; 25(1): 103-113.

28. Zarrazquin I, Torres Unda J, Ruiz F, Irazusta J, Maider KI, Hoyos CJ, Irazusta A. Longitudinal study: lifestyle and cardiovascular health in health science students. Nutr Hosp. 2014; 30(5): 11441151. DOI: $10.3305 /$ nh.2014.30.5.7833. 